\title{
Metabolic Abnormalities Rather Than BMI, Associated With Increased Risk of Recurrent Stroke in Chinese Hospitalized Stroke Patients: A Retrospective Study
}

\author{
Xiaolin Huang \\ the Third Affiliated Hospital of Soochow University \\ Jiaojiao Zhou \\ the Third Affiliated Hospital of Soochow University \\ Hong Zhang \\ the Third Affiliated Hospital of Soochow University \\ Pei Gao \\ the Third Affiliated Hospital of Soochow University \\ Long Wang \\ the Third Affiliated Hospital of Soochow University \\ Yuting Yang \\ Jurong City Center for Diseases Control and Prevention \\ Xiaohong Jiang \\ the Third Affiliated Hospital of Soochow University \\ Lu Chen ( $\square$ cl2324@czfph.com ) \\ the Third Affiliated Hospital of Soochow University \\ Fei Hua \\ the Third Affiliated Hospital of Soochow University
}

\section{Research Article}

Keywords: Metabolic abnormalities, Body mass index, Obesity, Recurrent stroke.

Posted Date: June 9th, 2021

DOI: https://doi.org/10.21203/rs.3.rs-579117/v1

License: (c) (i) This work is licensed under a Creative Commons Attribution 4.0 International License. Read Full License 


\section{Abstract \\ Background}

Metabolic abnormalities and body mass index (BMI) are known as apparent risk factors of recurrent stroke, but which one is more likely related to recurrent stroke remains uncertain. This study aimed to compare the metabolic phenotypes and $\mathrm{BMI}$ as indicators of recurrent stroke in Chinese hospitalized stroke patients.

\section{Methods}

In this retrospective population-based study, 856 hospitalized stroke patients from the Third Affiliated Hospital of Soochow University were enrolled. Recurrent stroke was defined as newly-onset stroke patients with a history of previous stroke. Metabolic phenotypes were categorized based on Adult Treatment Panel III criteria. BMI $\geq 25 \mathrm{~kg} / \mathrm{m}^{2}$ was defined as obesity.

\section{Results}

Among the hospitalized stroke patients, the prevalence of recurrent stroke was $21.9 \%$. Metabolic abnormalities rather than BMI were significantly associated with recurrent stroke. Compared with metabolically healthy patients, metabolically unhealthy ones had $72 \%$ (odds ratio [OR] $=1.72,95 \%$ confidence interval $[\mathrm{Cl}]$ 1.01-2.68) increased risk of recurrent stroke, regardless of $\mathrm{BMI}$ and other confounding factors. Whereas, no statistical association between BMI and recurrent stroke were found. Metabolic status significantly improved risk prediction of recurrent stroke when adding to the conventional-risk-factor model (net reclassification index 17.6\%, $\mathrm{P}=0.0047$; integrated discrimination improvement $0.7 \%, P=0.014$ ), while BMI did not.

\section{Conclusions}

Recurrent stroke is likely associated with metabolic abnormalities rather than with BMI. For the secondary prevention of stroke, controlling metabolic abnormalities is a more crucial method then BMI controlling in stroke patients.

\section{Introduction}

Obesity as a worldwide epidemic has emerged as a critical public health issue, for its considerable impact on a number of diseases such as stroke, cardiovascular diseases, diabetes, hypertension, and cancer ${ }^{1}$. Abundant evidence have suggested that obesity is also a risk factor for stroke for the primary prevention, overweight and obesity is associated with an overt increasing risk of stroke ${ }^{2}$, and a significant $6 \%$ 
increase was concomitant with each unit increase of body mass index (BMI $)^{3}$. However, for the secondary prevention, some prospective studies reported that obese patients after stroke had a lower risk of recurrent stroke $\mathrm{e}^{4}$, overweight and mild obese patients had better 10-year survival after stoke, compared with normal weight patients ${ }^{5}$. The association between obesity and recurrent stroke is undetermined. Unfavorable metabolic status probably contributes to the distinctive impact on recurrent stroke between obese and non-obese stroke patients ${ }^{6}$.

Metabolic syndrome (MetS) as an integrated statue of metabolic disorders-elevated plasma glycemia, central obesity, hypertension and dyslipidemia, is an important predictor of recurrent stroke, with $52 \%$ increased risk of recurrent stroke ${ }^{7}$. However, among the components of Mets, obesity illustrates lower influence than the other risk factors, no matter on stroke ${ }^{8}$ or recurrent stroke $e^{9,10}$. Concretely, a metaanalysis of cohort studies showed that the hazard ratio of obesity for recurrent stroke was 1.12 (0.891.41), significantly lower than that of elevated glycemia and dyslipidemia ${ }^{9}$. A multiclinic study among mild-to-moderate stroke patients also did not find the significant association of BMI with 5-year risk of recurrent stroke, which was lower than that of elevated blood pressure, elevated glycemia and low HDL$c^{11}$. Therefore, we assume that recurrent stroke probably be influenced by obesity in conjunction with the metabolic status.

Whether or not recurrent stroke is more likely related to poor metabolic health rather than to obesity remains uncertain, while conflicting data are similarly examined among the new-onset stroke. Previous studies reported that obese individuals with otherwise metabolically healthy profile, referred as "metabolically healthy obesity (MHO)", did not have increased risk of stroke than metabolically healthy

non-obesity ${ }^{10}$. However, apparent lean but metabolically unhealthy experienced higher risk of stroke, and the risk increased as the number of metabolic syndrome components increased ${ }^{12}$.

Thus, the prevent study aimed to compare the metabolic phenotypes and BMI as indicators of recurrent stroke in Chinese hospitalized stroke patients, and proposed the hypothesis that metabolic unhealthy status is more closely associated with the higher risk of recurrent stoke than obesity.

\section{Materials And Methods}

\section{Study population}

This retrospective study was conducted among the hospitalized patients who were diagnosed with cerebrovascular diseases in the Third Affiliated Hospital of Soochow University. From January 2017 to January 2018, a total of 870 participants were recruited with the inclusion criteria: 1) who agreed to participate the present study with the informed form; 2 ) the cerebrovascular diseases were diagnosed according to computed tomography (CT) or magnetic resonance imaging (MRI) scans. Each patient underwent a face-to-face interview for a standard questionnaire and blood samples collection for biochemical measurements. For present analysis, the exclusive criteria were: 1) patients with subarachnoid hemorrhage, transient ischemic attack without progression to stroke or lacunar infarction 
$(n=7) ;(2)$ missing information on lipid profiles, blood pressure and plasma glucose $(n=4)$; (3) extreme value of body mass index (BMI) $(n=3)$. Eventually, 856 participants were enrolled in present study.

The study protocol was approved by the Ethics Committee of the Third Affiliated Hospital of Soochow University. Each participants provided the written informed consent.

\section{Data collection}

A face-to-face interview was performed through a standard questionnaire for the collection of information on sociodemographic characteristics, lifestyle and medical history. Current smokers and drinkers were defined as those who have smoked cigarettes and consumed alcohol in the past six months. Physical activity was divided into high physical activity or not, based on the International Physical Activity Questionnaire (IPAQ). Dietary habit was classified as omnivorous, vegetable activists, and meat activists, as well as the duration and frequency of consuming vegetables, fruits and dairy products were collected.

Anthropometric information of each participant including blood pressure, height and body weight was measured by trained personnel according to a standard protocol. Blood pressure was measured using an automated electric device (OMRON Model HEM-752 FUZZY, Omron Company, Dalian, China), on the nondominant arm. Two blood pressure measurements were taken with 1-minute interval, the mean of two values were defined as the available blood pressure in the current analysis. BMI was calculated as weight in kilogram divided by squared height in meters.

\section{Biochemical measurements}

After at least 10-hour fasting, blood samples of each participants were obtained for the measurements of fasting plasma glucose (FPG) and lipid profiles (triglyceride, TG; low-density lipoprotein cholesterol, LDLc; high-density lipoprotein cholesterol, HDL-c; total cholesterol, TC), which were measured by an autoanalyzer (AU-5800 Chemistry System, Beckman, USA).

\section{Definitions}

\section{Obesity and metabolic phenotypes}

Obesity phenotypes were defined as $\mathrm{BMI} \geq 25 \mathrm{~kg} / \mathrm{m}^{2}$ and non-obesity as $\mathrm{BMl}<25 \mathrm{~kg} / \mathrm{m}^{2}$ based on the BMI cut-point among Asian population ${ }^{13}$, which had been verified among Chinese population recently ${ }^{14,15}$.

According to Adult Treatment Panel III (ATP-III), the metabolic phenotypes were determined as: 1) elevated blood pressure: systolic blood pressure (SBP) $\geq 130 \mathrm{mmHg}$ and/or diastolic blood pressure (DBP) $\geq 90$ $\mathrm{mmHg}$, and/or taking of antihypertensive drugs; 2) elevated blood glucose: FPG $\geq 5.6 \mathrm{mmol} / \mathrm{l}$, and/or taking of antidiabetic drugs; 3 ) high TG: TG $\geq 1.7 \mathrm{mmol} /$, and/or taking lipid-lowing medication; 4) low HDL-C: HDL-c $<1.04 \mathrm{mmol} / /$ in men and $<1.29 \mathrm{mmol} / \mathrm{l}$ in women. Individuals with two or fewer of the previously mentioned items were considered as being in a metabolically healthy status. 
Crossing the obese and metabolic phenotypes, we defined 1) metabolically healthy non-obesity (MHNO) as $\mathrm{BMI}<25 \mathrm{~kg} / \mathrm{m}^{2}$ and metabolically healthy status, 2) metabolically healthy obesity (MHO) as $\mathrm{BMI} \geq 25 \mathrm{~kg} / \mathrm{m}^{2}$ and metabolically healthy status, 3 ) metabolically unhealthy non-obesity (MUNO) as BMI $<25 \mathrm{~kg} / \mathrm{m}^{2}$ and metabolically unhealthy status, 4) metabolically unhealthy obesity (MUO) as $\mathrm{BMI} \geq 25 \mathrm{~kg} / \mathrm{m}^{2}$ and metabolically unhealthy status.

\section{Recurrent stroke}

Among the participants having a history of previous stroke, those with newly-onset stroke diagnosed by neurologists based on clinical symptoms, physical signs, and CT/MRI suggestions were considered as recurrent stroke.

\section{Statistical analyses}

The current analyses were conducted based on the combined obesity and metabolic phenotypes. Mean \pm standard (SD) and median (interquartile range) were used as the normally distributed and skewed continuous variables. Numbers (proportions) were used as the categorical variables. One-way ANOVA for continuous variables and the Chi-square for categorical variables were performed for the comparisons among the four phenotypes; linear regression was used to analyze the trend across the four phenotypes.

Dependent on multivariate logistic regression analyses, odds ratios (ORs) and $95 \%$ confidence intervals (Cls) were presented as the risk of recurrent stroke across the variant BMI groups, ATP-III components, and metabolic phenotypes, as well as the four phenotypes across the obesity and metabolic status.

We calculated C statistics, net reclassification index (NRI), and integrated discrimination improvement (IDI) to evaluate the incremental prognostic values of individual $\mathrm{BMI} /$ metabolic status or the combined risk factors beyond conventional risk factors ${ }^{16}$.

We used SAS statistical software (version 9.3, Cary, NC) to perform all the statistical analyses, and considered two-tailed $P$ values $<0.05$ were statistically significant.

\section{Results}

\section{Characteristics of study population according to the obesity and metabolic phenotypes}

Sociodemographic, medical history and biochemical characteristics of the study population were presented in Table 1. Among all of the 856 participants, $44.8 \%(n=384)$ were obese and $77.7 \%(n=665)$ were metabolically unhealthy. The prevalence of different phenotypes were $14.5 \%$ for MHNO, $7.8 \%$ for MHO, $40.7 \%$ for MUNO, and $37.0 \%$ for MUO. Additionally, MHO accounted for $17.4 \%$ of obesity participants. Obese participants were younger, had higher blood pressure and serum lipids levels than non-obese individuals, no matter in metabolically healthy or unhealthy groups. Among the 
obesity/metabolic phenotypes, compared to the MHNO, individuals in MHO, MUNO, MUO were had gradually higher levels of TG, TC, LDL-C, FPG, proportions of hypertension, diabetes, hypercholesterolaemia, and higher usage of antihypertensive and antidiabetic drugs (all $P$ values for trend $<0.05$ ). 
Table 1

Baseline characteristics of participants according to metabolic and obesity phenotypes

\begin{tabular}{|c|c|c|c|c|c|}
\hline & \multicolumn{2}{|c|}{ Metabolically healthy } & \multicolumn{2}{|c|}{ Metabolically unhealthy } & \multirow{3}{*}{$\begin{array}{l}P \text { for } \\
\text { trend }\end{array}$} \\
\hline & $\begin{array}{l}\text { Non-obese } \\
\text { (MHNO) }\end{array}$ & $\begin{array}{l}\text { Obese } \\
\text { (MHO) }\end{array}$ & $\begin{array}{l}\text { Non-obese } \\
\text { (MUNO) }\end{array}$ & $\begin{array}{l}\text { Obese } \\
\text { (MUO) }\end{array}$ & \\
\hline & $(n=124)$ & $(n=67)$ & $(n=348)$ & $(n=317)$ & \\
\hline Male, n (\%) & $98(79.0)$ & $48(71.6)$ & $210(60.3)$ & $222(70.0)$ & 0.0928 \\
\hline Age (years) & $69.31 \pm 11.59$ & $\begin{array}{l}64.15 \pm \\
12.91\end{array}$ & $68.79 \pm 11.91$ & $\begin{array}{l}64.29 \pm \\
12.36\end{array}$ & 0.0007 \\
\hline $\mathrm{BMI}\left(\mathrm{kg} / \mathrm{m}^{2}\right)$ & $21.19 \pm 1.73$ & $\begin{array}{l}25.84 \pm \\
1.66\end{array}$ & $21.72 \pm 1.71$ & $\begin{array}{l}26.77 \pm \\
2.57\end{array}$ & $<.0001$ \\
\hline $\begin{array}{l}\text { High school education or } \\
\text { above, } \mathrm{n}(\%)\end{array}$ & $31(25.0)$ & $24(35.8)$ & $73(21.0)$ & $96(30.3)$ & 0.4674 \\
\hline $\begin{array}{l}\text { Annual income } \geq 10000 \\
\text { RMB, } n(\%)\end{array}$ & $103(83.1)$ & $64(95.5)$ & $281(80.8)$ & $260(82.0)$ & 0.2989 \\
\hline \multicolumn{6}{|l|}{ Lifestyle factors } \\
\hline Current smokers, n (\%) & $65(52.4)$ & $32(47.8)$ & $131(37.6)$ & $139(43.9)$ & 0.1041 \\
\hline Current drinkers, n (\%) & $44(35.5)$ & $23(34.3)$ & $84(24.1)$ & 99 (31.2) & 0.3509 \\
\hline $\begin{array}{l}\text { High physical activity, } \mathrm{n} \\
(\%)\end{array}$ & $43(34.7)$ & $24(35.8)$ & $128(36.8)$ & $102(32.2)$ & 0.5381 \\
\hline Healthy diet, n (\%) & $20(16.1)$ & $6(9.0)$ & $56(16.1)$ & $52(16.4)$ & 0.5978 \\
\hline \multicolumn{6}{|l|}{$\begin{array}{l}\text { Metabolism-related } \\
\text { measurements }\end{array}$} \\
\hline $\mathrm{SBP}(\mathrm{mmHg})$ & $\begin{array}{l}148.65 \pm \\
23.79\end{array}$ & $\begin{array}{l}146.85 \pm \\
21.94\end{array}$ & $\begin{array}{l}150.72 \pm \\
19.35\end{array}$ & $\begin{array}{l}151.63 \pm \\
17.88\end{array}$ & 0.0931 \\
\hline $\mathrm{DBP}(\mathrm{mmHg})$ & $83.27 \pm 13.36$ & $\begin{array}{l}85.52 \pm \\
10.62\end{array}$ & $82.88 \pm 12.06$ & $\begin{array}{l}86.51 \pm \\
12.86\end{array}$ & 0.0228 \\
\hline $\mathrm{TG}(\mathrm{mmol} / \mathrm{L})$ & $\begin{array}{l}1.07(0.83- \\
1.28)\end{array}$ & $\begin{array}{l}1.16(0.90- \\
1.46)\end{array}$ & $\begin{array}{l}1.56(1.16- \\
2.07)\end{array}$ & $\begin{array}{l}1.89(1.36- \\
2.66)\end{array}$ & $<.0001$ \\
\hline $\mathrm{TC}(\mathrm{mmol} / \mathrm{L})$ & $4.36 \pm 0.89$ & $4.54 \pm 0.82$ & $4.40 \pm 1.10$ & $4.67 \pm 1.05$ & 0.0061 \\
\hline $\mathrm{HDL}-\mathrm{C}(\mathrm{mmol} / \mathrm{L})$ & $1.43 \pm 0.51$ & $1.31 \pm 0.23$ & $1.06 \pm 0.30$ & $1.04 \pm 0.40$ & $<.0001$ \\
\hline
\end{tabular}

Data were means \pm SD or medians (interquartile ranges) for continuous variables, and numbers (percentages) for categorical variables. $P$ values were calculated from one-way ANOVA for continuous variables and $\chi^{2}$ test for categorical variables. Abbreviations: BMI, body mass index; SBP, systolic blood pressure; DBP, diastolic blood pressure; TC, total cholesterol; TG, triglycerides; HDL-C, highdensity lipoprotein cholesterol; LDL-C, low-density lipoprotein cholesterol; FPG, fasting plasma glucose; 


\begin{tabular}{|c|c|c|c|c|c|}
\hline & \multicolumn{2}{|c|}{ Metabolically healthy } & \multicolumn{2}{|c|}{ Metabolically unhealthy } & \multirow{3}{*}{$\begin{array}{l}P \text { for } \\
\text { trend }\end{array}$} \\
\hline & $\begin{array}{l}\text { Non-obese } \\
\text { (MHNO) }\end{array}$ & $\begin{array}{l}\text { Obese } \\
\text { (MHO) }\end{array}$ & $\begin{array}{l}\text { Non-obese } \\
\text { (MUNO) }\end{array}$ & $\begin{array}{l}\text { Obese } \\
\text { (MUO) }\end{array}$ & \\
\hline & $(n=124)$ & $(n=67)$ & $(n=348)$ & $(n=317)$ & \\
\hline LDL-C (mmol/L) & $2.26 \pm 0.57$ & $2.39 \pm 0.66$ & $2.36 \pm 0.78$ & $2.58 \pm 0.88$ & $<.0001$ \\
\hline FPG (mmol/L) & $\begin{array}{l}4.94(4.52- \\
5.43)\end{array}$ & $\begin{array}{l}4.90(4.44- \\
5.40)\end{array}$ & $\begin{array}{l}5.90(4.92- \\
7.59)\end{array}$ & $\begin{array}{l}6.10(5.10- \\
7.66)\end{array}$ & $<.0001$ \\
\hline \multicolumn{6}{|l|}{ Diseases, n (\%) } \\
\hline Hypertension, n (\%) & $90(72.6)$ & $49(73.1)$ & $305(87.6)$ & $287(90.5)$ & $<.0001$ \\
\hline Diabetes, n (\%) & $1(0.8)$ & $1(1.5)$ & $153(44.0)$ & $140(44.2)$ & $<.0001$ \\
\hline $\begin{array}{l}\text { Hypercholesterolaemia, n } \\
(\%)\end{array}$ & $1(0.8)$ & $2(3.0)$ & $13(3.7)$ & $23(7.3)$ & 0.0022 \\
\hline \multicolumn{6}{|l|}{ Drug use, $\mathrm{n}(\%)$} \\
\hline Antihypertensive drug use & 48 (38.7) & $31(46.3)$ & $221(63.5)$ & $210(66.3)$ & $<.0001$ \\
\hline Antidiabetic drug use & $0(0.0)$ & $0(0.0)$ & $87(25.0)$ & $77(24.3)$ & $<.0001$ \\
\hline Lipid-lowering drug use & $1(0.8)$ & $0(0.0)$ & $8(2.3)$ & $8(2.5)$ & 0.1498 \\
\hline \multicolumn{6}{|c|}{$\begin{array}{l}\text { Data were means } \pm \text { SD or medians (interquartile ranges) for continuous variables, and numbers } \\
\text { (percentages) for categorical variables. P values were calculated from one-way ANOVA for continuous } \\
\text { variables and } \chi^{2} \text { test for categorical variables. Abbreviations: BMI, body mass index; SBP, systolic } \\
\text { blood pressure; DBP, diastolic blood pressure; TC, total cholesterol; TG, triglycerides; HDL-C, high- } \\
\text { density lipoprotein cholesterol; LDL-C, low-density lipoprotein cholesterol; FPG, fasting plasma } \\
\text { glucose; }\end{array}$} \\
\hline
\end{tabular}

\section{Obesity/metabolic Phenotypes And Risk Of Recurrent Stroke}

Table 2 showed the obesity/metabolic phenotypes and risk of recurrent stroke using multiple logistic regression. Divided BMI into two categories, comparing to non-obesity, the risk of recurrent stroke increased $50 \%(\mathrm{OR}=1.50,95 \% \mathrm{Cl} 1.06-2.13)$ in obese individuals after adjusting for age, sex, lifestyle, education, income, serum lipids, blood pressure, glucose, medication and family history. However, with the increase of BMI, significantly increased risk of recurrent stroke was not found. Among the ATP- $\mathbb{X}$ components, elevated blood pressure and glucose and low HDL-c, but not high TG, were associated with increased risk of recurrent stroke in the multivariate-adjusted model $(\mathrm{OR}=3.55,95 \% \mathrm{Cl} 1.33-7.93$ for elevated blood pressure and $\mathrm{OR}=1.6295 \% \mathrm{Cl} 1.14-2.28$ for elevated blood glucose and $\mathrm{OR}=1.4695 \% \mathrm{Cl}$ 1.01-2.11 for low HDL-c). In terms of metabolic status, referred to metabolically healthy, metabolically unhealthy individuals had $72 \%(\mathrm{OR}=1.72,95 \% \mathrm{Cl} 1.01-2.68)$ increased risk of recurrent stroke. 
Table 2

Risk of prevalent recurrent stroke according to BMI, ATP- $\$ components and metabolic status

Model 1

Model 2

\section{obesity $^{a}$}

Non-obesity, $\mathrm{BMI}<25 \mathrm{~kg} / \mathrm{m}^{2}$

1.00 (Ref)

1.00 (Ref)

Obesity, $\mathrm{BMI} \geq 25 \mathrm{~kg} / \mathrm{m}^{2}$

$1.31(0.95-1.80)$

$1.50(1.06-2.13)$

$\mathrm{BMI}, 3.26 \mathrm{~kg} / \mathrm{m}^{2}$

$1.03(0.88-1.21)$

$1.09(0.92-1.30)$

\section{ATP-\ components ${ }^{b}$}

$\mathrm{BP} \geq 130 / 85 \mathrm{mmHg}$ or on medication

$3.08(1.31-7.23)$

$3.25(1.33-7.93)$

FPG $\geq 6.1 \mathrm{mmol} / \mathrm{L}$ or on medication

$1.77(1.28-2.44)$

$1.62(1.14-2.28)$

$\mathrm{TG} \geq 1.7 \mathrm{mmol} / \mathrm{L}$ or on medication

$0.83(0.59-1.15)$

$0.75(0.52-1.10)$

$\mathrm{HDL}-\mathrm{c}<1.04 \mathrm{mmol} / \mathrm{L}(<1.29 \mathrm{mmol} / \mathrm{L}$ in women)

$1.59(1.13-2.23)$

$1.46(1.01-2.11)$

\section{Metabolic status $^{c}$}

Metabolically healthy

1.00 (Ref)

1.00 (Ref)

Metabolically unhealthy

$1.81(1.18-2.80)$

$1.72(1.10-2.68)$

Model 1: Unadjusted;

Model 2: Adjusted for age, sex, current smokers (yes/no), current drinking (yes/no), physical activity, diet, education, income, and ${ }^{\mathrm{a}} \mathrm{TG}, \mathrm{HDL}-\mathrm{c}, \mathrm{SBP}, \mathrm{FPG}$, use of antihypertensive/ antidiabetic/ antilipemic drugs, and family history of hypertension/diabetes/hyperlipemia, or ${ }^{\mathrm{b}} \mathrm{BMI}$ and other ATP- $\bigotimes$

components, or ${ }^{\mathrm{B}} \mathrm{BMI}$.

Crossing the obesity and metabolic phenotypes, we reclassified the participants into four groups: MHNO, MHO, MUNO, and MUO. The prevalence of recurrent stroke was 16.1\% (20/124) in MHNO, 13.4\% (9/67) in MHO, 21.8\% (76/348) in MUNO, and 27\% (87/317) in MUO. The multivariate-adjusted logistic regression (Fig. 1) did not find the significant association between $\mathrm{MHO}$ and recurrent stroke, comparing to the nonobese counterparts. Likewise, individuals with MUNO did not have higher risk of recurrent stroke compared to MHNO. However, when combining the obesity and metabolic status, 1.14-fold increased risk of recurrent stroke was found in MUO compared to MHNO.

\section{Incremental Prognostic Values Of Bmi/metabolic Status}

Table 3 showed whether individual BMI or metabolic status could add the prognostic value of recurrent stroke to the conventional risk factors. The $\mathrm{C}$ statistic $(95 \% \mathrm{Cl})$ of the predictive models of conventional risk factors was $0.607(0.574-0.640)$, and the addition of $\mathrm{BMI}$ or metabolic status did not increase the $\mathrm{C}$ 
statistic. Nevertheless, we found metabolic status, but not BMI, could significantly improve reclassification risk of recurrent stroke when adding to the conventional model. Adding the combined BMI and metabolic status to the conventional model, greater incremental prognostic values were found (all $P$ values<0.05).

Table 3

Improvement in predicting recurrent stroke by adding BMI and metabolic status to conventional risk factors

\begin{tabular}{|c|c|c|c|c|c|c|}
\hline & \multicolumn{2}{|l|}{ C statistic } & \multicolumn{2}{|c|}{ NRI (continuous) } & \multicolumn{2}{|l|}{ IDI } \\
\hline & $\begin{array}{l}\text { Estimate } \\
(95 \% \mathrm{Cl})\end{array}$ & $\begin{array}{l}P \\
\text { value }^{b}\end{array}$ & $\begin{array}{l}\text { Estimate } \\
(95 \% \mathrm{Cl})\end{array}$ & $\begin{array}{l}P \\
\text { value }^{b}\end{array}$ & $\begin{array}{l}\text { Estimate } \\
(95 \% \mathrm{Cl})\end{array}$ & $\begin{array}{l}P \\
\text { value }\end{array}$ \\
\hline Conventional model ${ }^{\mathrm{a}}$ & $\begin{array}{l}0.607 \\
(0.574- \\
0.640)\end{array}$ & - & Reference & - & Reference & - \\
\hline Conventional model + BMI & $\begin{array}{l}0.608 \\
(0.574- \\
0.641)\end{array}$ & 0.89 & $\begin{array}{l}0.139 \\
(-0.021- \\
0.299)\end{array}$ & 0.089 & $\begin{array}{l}0.0029 \\
(-0.0005- \\
0.0064)\end{array}$ & 0.095 \\
\hline $\begin{array}{l}\text { Conventional model + } \\
\text { Metabolic status }\end{array}$ & $\begin{array}{l}0.624 \\
(0.590- \\
0.656)\end{array}$ & 0.19 & $\begin{array}{l}0.176 \\
(0.054- \\
0.297)\end{array}$ & 0.0047 & $\begin{array}{l}0.007 \\
(0.0014- \\
0.0127)\end{array}$ & 0.014 \\
\hline $\begin{array}{l}\text { Conventional model + BMI } \\
\text { and Metabolic status }\end{array}$ & $\begin{array}{l}0.624 \\
(0.591- \\
0.657)\end{array}$ & 0.17 & $\begin{array}{l}0.195 \\
(0.058- \\
0.332)\end{array}$ & 0.0052 & $\begin{array}{l}0.009 \\
(0.0025- \\
0.0148)\end{array}$ & 0.0059 \\
\hline \multicolumn{7}{|c|}{$\begin{array}{l}\text { aC Conventional risk factors including age, sex, current smokers (yes/no), current drinking (yes/no), } \\
\text { physical activity, diet, education and income. }\end{array}$} \\
\hline \multicolumn{7}{|c|}{$\mathbf{b}_{P}$ values were compared to the conventional model. } \\
\hline \multicolumn{7}{|c|}{$\begin{array}{l}\text { Abbreviations: BMI, body mass index; NRI, net reclassification index; IDI, integrated discrimination } \\
\text { improvement; } \mathrm{Cl} \text {, confidence interval. }\end{array}$} \\
\hline
\end{tabular}

\section{Discussion}

In the present study, we found that among the Chinese hospitalized stroke patients, metabolically

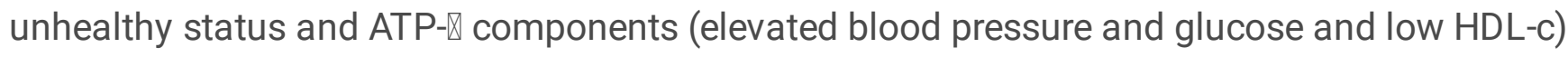
significantly increase the risk of recurrent stroke, regardless of BMI, whereas no association existed between $\mathrm{BMI}$ and risk of recurrent stroke. Furthermore, we found metabolic status, but not BMI, significantly improved prognostic value of recurrent stroke when adding to the conventional risk factors.

Numerous previous studies have demonstrated metabolic syndrome was associated with increased risk of recurrent stroke ${ }^{11,17,18}$, the ACROSS-China study showing a hazard ratio of 1.94 (1.39-2.73) in patients with metabolic syndrome vs. no metabolic syndrome ${ }^{18}$, a longitudinal study calculating a higher risk of recurrent stroke with the hazard ratio of $1.43(1.01-2.01)^{11}$. Further controlling for its components, 
the significant associations disappeared. In accordance with precious studies, our study found metabolic unhealthy individuals had $72 \%$ higher risk of recurrent stroke than metabolic healthy ones, as well as its components (elevated blood pressure and fasting glucose and low HDL-c levels). All these results underlined the crucial value of metabolic status on stroke prognosis.

Although obesity is a considerable risk factor of stroke, no matter defined based on waist circumference or BMI, some certain studies interestingly reported that obesity might be a "protective" factor of stroke recurrence $4,19,20$. The obesity paradox is partly attributed to the selection bias (obesity patients are younger, more likely to be female and have less proportion of smokers) and the limitation of obesity definition ${ }^{20}$. Inconsistent with the situation aforementioned, the present study detected that obesity patients (defined as $\mathrm{BMI} \geq 25 \mathrm{~kg} / \mathrm{m}^{2}$ ), controlling metabolic confounder factors, had weakly higher risk of recurrent stroke. Howbeit $\mathrm{BMI}$, as a continuous variable, was not found associated with recurrent stroke. The discrepancies might be attributed to the indiscriminate gender proportion $(P=0.42)$ and lifestyle $(P$ for current smoker was $0.60, P$ for current drinker was 0.07 ) between obesity and non-obesity individuals, and further adjusting metabolic-related confounders, although the obesity patients were younger $(P<$ $0.001)$.

This is the first study to investigate and compare the effects of metabolic status and BMI on recurrent stroke. In order to confirm the impact of metabolic status and BMI, we stratified the population based on metabolic phenotypes and different BMI group (BMI $\geq 25 \mathrm{~kg} / \mathrm{m}^{2}$ and $\left.\mathrm{BMI}<25 \mathrm{~kg} / \mathrm{m}^{2}\right)$ into four groups: MHNO, MHO, MUNO and MUO groups. Previous data about the obesity/metabolic phenotypes and stroke were focused on the stroke primary prevention field, and drawn controversial conclusions ${ }^{21-24}$. A nationwide population-based study in Korea reported that $\mathrm{MHO}$ individuals were not at increased risk of stroke ${ }^{21}$, whereas another longitude study in 7122 participants found that $\mathrm{MHO}$ individuals were at increased risk of cardiovascular disease including stroke, and stroke separately ${ }^{22}$. Studies about the effect of obesity/metabolic phenotypes on stroke prognosis were sparsely. Results in present study showed that, compared to MHNO, MUO had statistically higher risk of recurrent stroke, and the OR of MUNO was higher than that of MHO, although the $P$ values of ORs were less than 0.05 . Above-mentioned results suggested that metabolic status, but not BMI per se, increased the risk of recurrent stroke. Furthermore, evidenced by NRI and IDI, metabolic phenotypes rather than BMI were associated with higher stroke recurrence risks, adding metabolic status to conventional model substantially improved the risk stratification for recurrent stroke, while adding BMI did not.

Several suggested mechanisms could interpret metabolic status is more important than BMI in risk of recurrent stroke. Firstly, chronic inflammation was postulated to initiate recurrent stroke in metabolically unhealthy stroke patients, regardless of BMI. It has been well known that inflammation plays a critical role, through mechanisms such as the development of atherosclerosis, plaque instability, and triggering of plaque rupture, in the pathogenesis of cerebrovascular diseases ${ }^{25}$. Sufficient population-based cohort studies similarly demonstrated the most extensively used blood marker of inflammation, C-reactive protein (CRP), significantly contributed to the recurrent stroke $e^{26-28}$. Recently, several previous studies 
reported metabolically unhealthy obese and nonobese individuals had unfavorable inflammation status than metabolically healthy counterparts ${ }^{29-31}$, with higher level of C-reactive protein, IL-6, tumor necrosis factor $a(T N F-a)$, adipocyte fatty acid binding protein (A-FABP). Thus, the unfavorable inflammation status of metabolically unhealthy individuals, in all likelihood, mediated recurrent stroke. In addition, visceral adipose tissue may also play a substantial role. Inflammation of visceral adipose tissue mediated metabolically unhealthy status regardless of generalized obesity ${ }^{32}$. Camhi SM, et al. also revealed that metabolically unhealthy individuals, no matter obese or non-obese, had higher amount of visceral adiposity or liver fat than metabolically healthy participants ${ }^{33}$. Visceral adiposity, suggested by sufficient evidence, was a risk factor for recurrent stroke ${ }^{7,34}$. A retrospective study in Japan found that recurrent ischemic stroke patients had significantly higher visceral fat level than those without recurrence, and visceral fat level was associated with risk of recurrent stroke $(\mathrm{OR}=2.44,95 \% \mathrm{Cl} 1.08-5.49)^{34}$.

The present study had a few limitations of note. Firstly, medications which may affect the results were not fully adjusted. However, we had adjusted the antihypertensive/antidiabetic drugs and lipid-lowering drugs, which may have great influence on the development of atherosclerosis and plaque instability, in the multivariate logistic regression. Secondly, our study was a retrospective study, a prospective study was warranted to further definite the associations of metabolic phenotypes and BMI with recurrent stroke. Thirdly, our present study was performed in Chinese hospital patients, it should be cautious to generalize our findings to other ethnicities.

\section{Conclusions}

In conclusion, our present study found a significantly association of increased recurrent stroke risk with metabolically abnormalities, while the association between BMI and recurrent stroke was not detected. In addition, adding to the conventional risk factors, metabolic status statistically improved prognostic value of recurrent stroke, whereas BMI did not. It is suggested that recurrent stroke is, to a large extent, related to poor metabolic status rather than to BMI. Therefore, metabolic abnormalities controlling could be a more efficacious method to prevent recurrent stroke than weight controlling in stroke patients.

\section{List Of Abbreviations}

ATP-III, Adult Treatment Panel III; A-FABP, adipocyte fatty acid binding protein; BMI, Body mass index; CT, computed tomography; $\mathrm{Cl}$, confidence intervals; CRP, C-reactive protein; DBP, diastolic blood pressure; FPG, fasting plasma glucose; HDL-c, high-density lipoprotein cholesterol; IDI, integrated discrimination improvement; IPAQ, International Physical Activity Questionnaire; LDL-c, low-density lipoprotein cholesterol; Mets, Metabolic syndrome; MHO, Metabolically healthy obesity; MHNO, metabolically healthy non-obesity; MUNO, metabolically unhealthy non-obesity; MUO, metabolically unhealthy obesity; MRI, magnetic resonance imaging; NRI, net reclassification index; OR, odds ratios; SBP, systolic blood pressure; SD, standard; TG, triglyceride; TC, total cholesterol; TNF-a, tumor necrosis factor a. 


\section{Declarations}

\section{Ethics approval and consent to participate}

The study protocol was approved by the Ethics Committee of the Third Affiliated Hospital of Soochow University. Each participants provided the written informed consent.

\section{Consent for publication}

Not applicable.

Availability of data and materials: The data in the current study arisen from a dataset of Department of Endocrine and Metabolic Diseases, the Third Affiliated Hospital of Soochow University, are not publicly available due to security consideration, but are available from the corresponding author on reasonable request.

Competing interests: The authors declare that they have no competing interests.

Funding: This study was supported by the grants 81900768 from the National Natural Science Foundation of China, CJ20190103 from the Applied and Basic Research Program of Changzhou Scientific and Technological Project.

\section{Authors' contributions}

$\mathrm{LC}$ and $\mathrm{FH}$ contributed to the conception and design of this study. $\mathrm{XH}, \mathrm{JZ}, \mathrm{HZ}, \mathrm{PG}, \mathrm{LW}, \mathrm{YY}$ and $\mathrm{XJ}$ were involved in the acquisition of data. $\mathrm{XH}, \mathrm{JZ}$ and $\mathrm{HZ}$ conducted the statistical analysis and interpretation of data, and drafted the manuscript. XJ, LW, LC and FH contributed to the revision of manuscript for important intellectual content. All authors read and approved the final manuscript.

Acknowledgments: We thank for all the participants for their participation and clinical colleagues for their valuable assistance.

\section{Authors' information}

${ }^{1}$ Department of Endocrine and Metabolic Diseases, the Third Affiliated Hospital of Soochow University, Changzhou 213000, Jiangsu, China. ${ }^{2}$ Department of Endocrinology, Jiangsu University Affiliated Wujin Hospital, Changzhou 213000, Jiangsu, China. ${ }^{3}$ Jurong City Center for Diseases Control and Prevention, Jurong 212400, Jiangsu, China. ${ }^{4}$ Department of Clinical Nutrition, the Third Affiliated Hospital of Soochow University, Changzhou 213000, Jiangsu, China.

\section{References}

1. Poirier P, Giles TD, Bray GA, Hong Y, Stern JS, Pi-Sunyer FX, et al. Obesity and cardiovascular disease: pathophysiology, evaluation, and effect of weight loss: an update of the 1997 American Heart 
Association Scientific Statement on Obesity and Heart Disease from the Obesity Committee of the Council on Nutrition, Physical Activity, and Metabolism. Circulation. 2006;113(6):898-918.

2. Strazzullo P, D'Elia L, Cairella G, Garbagnati F, Cappuccio FP, Scalfi L. Excess body weight and incidence of stroke: meta-analysis of prospective studies with 2 million participants. Stroke. 2010;41(5):e418-26.

3. Kurth T, Gaziano JM, Berger K, Kase CS, Rexrode KM, Cook NR, et al. Body mass index and the risk of stroke in men. Arch Intern Med. 2002;162(22):2557-62.

4. Doehner W, Schenkel J, Anker SD, Springer J, Audebert HJ. Overweight and obesity are associated with improved survival, functional outcome, and stroke recurrence after acute stroke or transient ischaemic attack: observations from the TEMPiS trial. Eur Heart J. 2013;34(4):268-77.

5. Aparicio HJ, Himali JJ, Beiser AS, Davis-Plourde KL, Vasan RS, Kase CS, et al. Overweight, Obesity, and Survival After Stroke in the Framingham Heart Study. J Am Heart Assoc. 2017;6(6).

6. Oesch L, Tatlisumak T, Arnold M, Sarikaya H. Obesity paradox in stroke - Myth or reality? A systematic review. PLoS One. 2017;12(3):e0171334.

7. Chen W, Pan Y, Jing J, Zhao X, Liu L, Meng X, et al. Recurrent Stroke in Minor Ischemic Stroke or Transient Ischemic Attack With Metabolic Syndrome and/or Diabetes Mellitus. J Am Heart Assoc. 2017;6(6).

8. Byun AR, Kwon S, Lee SW, Shim KW, Lee HS. Metabolic health is more closely associated with prevalence of cardiovascular diseases or stroke than obesity: A cross-sectional study in Korean populations. Medicine (Baltimore). 2016;95(24):e3902.

9. Li X, Li X, Fang F, Fu X, Lin H, Gao Q. Is Metabolic Syndrome Associated with the Risk of Recurrent Stroke: A Meta-Analysis of Cohort Studies. J Stroke Cerebrovasc Dis. 2017;26(12):2700-5.

10. Li Z, Guo X, Liu Y, Zhang N, Chang Y, Chen Y, et al. Metabolism rather than obesity is associated with ischemic stroke: a cross-sectional study in rural Northeastern China. Springerplus. 2016;5(1):1419.

11. Fang X, Liu H, Zhang X, Zhang H, Qin X, Ji X. Metabolic syndrome, its components, and diabetes on 5-year risk of recurrent stroke among mild-to-moderate ischemic stroke survivors: a multiclinic registry study. Journal of Stroke and Cerebrovascular Diseases. 2016;25(3):626-34.

12. Seo YG, Choi HC, Cho B. The Relationship between Metabolically Obese Non-Obese Weight and Stroke: The Korea National Health and Nutrition Examination Survey. PLoS One. 2016;11(8):e0160846.

13. Appropriate body-mass index for Asian populations and its implications for policy and intervention strategies. Lancet. 2004;363(9403):157-63.

14. Lin L, Zhang J, Jiang L, Du R, Hu C, Lu J, et al. Transition of metabolic phenotypes and risk of subclinical atherosclerosis according to BMI: a prospective study. Diabetologia. 2020;63(7):1312-23.

15. Lin L, Peng K, Du R, Huang X, Lu J, Xu Y, et al. Metabolically healthy obesity and incident chronic kidney disease: The role of systemic inflammation in a prospective study. Obesity (Silver Spring). 2017;25(3):634-41. 
16. Pencina MJ, D'Agostino RB, Pencina KM, Janssens AC, Greenland P. Interpreting incremental value of markers added to risk prediction models. Am J Epidemiol. 2012;176(6):473-81.

17. Liu L, Zhan L, Wang Y, Bai C, Guo J, Lin Q, et al. Metabolic syndrome and the short-term prognosis of acute ischemic stroke: a hospital-based retrospective study. Lipids Health Dis. 2015;14:76.

18. Mi D, Jia Q, Zheng H, Hoff K, Zhao X, Wang C, et al. Metabolic syndrome and stroke recurrence in Chinese ischemic stroke patients--the ACROSS-China study. PLoS One. 2012;7(12):e51406.

19. Zhao L, Du W, Zhao X, Liu L, Wang C, Wang Y, et al. Favorable functional recovery in overweight ischemic stroke survivors: findings from the China National Stroke Registry. J Stroke Cerebrovasc Dis. 2014;23(3):e201-6.

20. Ovbiagele B, Bath PM, Cotton D, Vinisko R, Diener HC. Obesity and recurrent vascular risk after a recent ischemic stroke. Stroke. 2011;42(12):3397-402.

21. Lee HJ, Choi EK, Lee SH, Kim YJ, Han KD, Oh S. Risk of ischemic stroke in metabolically healthy obesity: A nationwide population-based study. PLoS One. 2018;13(3):e0195210.

22. Hinnouho GM, Czernichow S, Dugravot A, Nabi H, Brunner EJ, Kivimaki M, et al. Metabolically healthy obesity and the risk of cardiovascular disease and type 2 diabetes: the Whitehall II cohort study. Eur Heart J. 2015;36(9):551-9.

23. Aung K, Lorenzo C, Hinojosa MA, Haffner SM. Risk of developing diabetes and cardiovascular disease in metabolically unhealthy normal-weight and metabolically healthy obese individuals. J Clin Endocrinol Metab. 2014;99(2):462-8.

24. Sánchez-Iñigo L, Navarro-González D, Fernández-Montero A, Pastrana-Delgado J, Martínez JA. Risk of incident ischemic stroke according to the metabolic health and obesity states in the VascularMetabolic CUN cohort. Int J Stroke. 2017;12(2):187-91.

25. Di Napoli M, Elkind MS, Godoy DA, Singh P, Papa F, Popa-Wagner A. Role of C-reactive protein in cerebrovascular disease: a critical review. Expert Rev Cardiovasc Ther. 2011;9(12):1565-84.

26. Kitagawa K, Hosomi N, Nagai Y, Kagimura T, Ohtsuki T, Maruyama H, et al. Cumulative Effects of LDL Cholesterol and CRP Levels on Recurrent Stroke and TIA. J Atheroscler Thromb. 2019;26(5):432-41.

27. Matsuo R, Ago T, Hata J, Wakisaka Y, Kuroda J, Kuwashiro T, et al. Plasma C-Reactive Protein and Clinical Outcomes after Acute Ischemic Stroke: A Prospective Observational Study. PLoS One. 2016;11(6):e0156790.

28. Liu H, Qian S, Zhong C, Wang A, Peng Y, Peng H, et al. Predictive Value of Cystatin C for Stroke Recurrence in Patients With Acute Ischemic Stroke. Circ J. 2021;85(2):213-9.

29. Graf C, Ferrari N. Metabolic Health-The Role of Adipo-Myokines. Int J Mol Sci. 2019;20(24).

30. Lee TH, Jeon WS, Han KJ, Lee SY, Kim NH, Chae HB, et al. Comparison of Serum Adipocytokine Levels according to Metabolic Health and Obesity Status. Endocrinol Metab (Seoul). 2015;30(2):18594.

31. Phillips CM, Perry IJ. Does Inflammation Determine Metabolic Health Status in Obese and Nonobese Adults? The Journal of Clinical Endocrinology \& Metabolism. 2013;98(10):E1610-E9. 
32. Blüher M. The distinction of metabolically 'healthy' from 'unhealthy' obese individuals. Curr Opin Lipidol. 2010;21(1):38-43.

33. Camhi SM, Katzmarzyk PT. Differences in body composition between metabolically healthy obese and metabolically abnormal obese adults. Int J Obes (Lond). 2014;38(8):1142-5.

34. Ushio M, Kanaoka M, Kinoshita Y, Maeno S, Fujita K. Moderate-to-vigorous physical activity and the risk of stroke recurrence in patients with a history of minor ischemic stroke in Japan: a retrospective analysis. Top Stroke Rehabil. 2018;25(8):591-8.

\section{Figures}

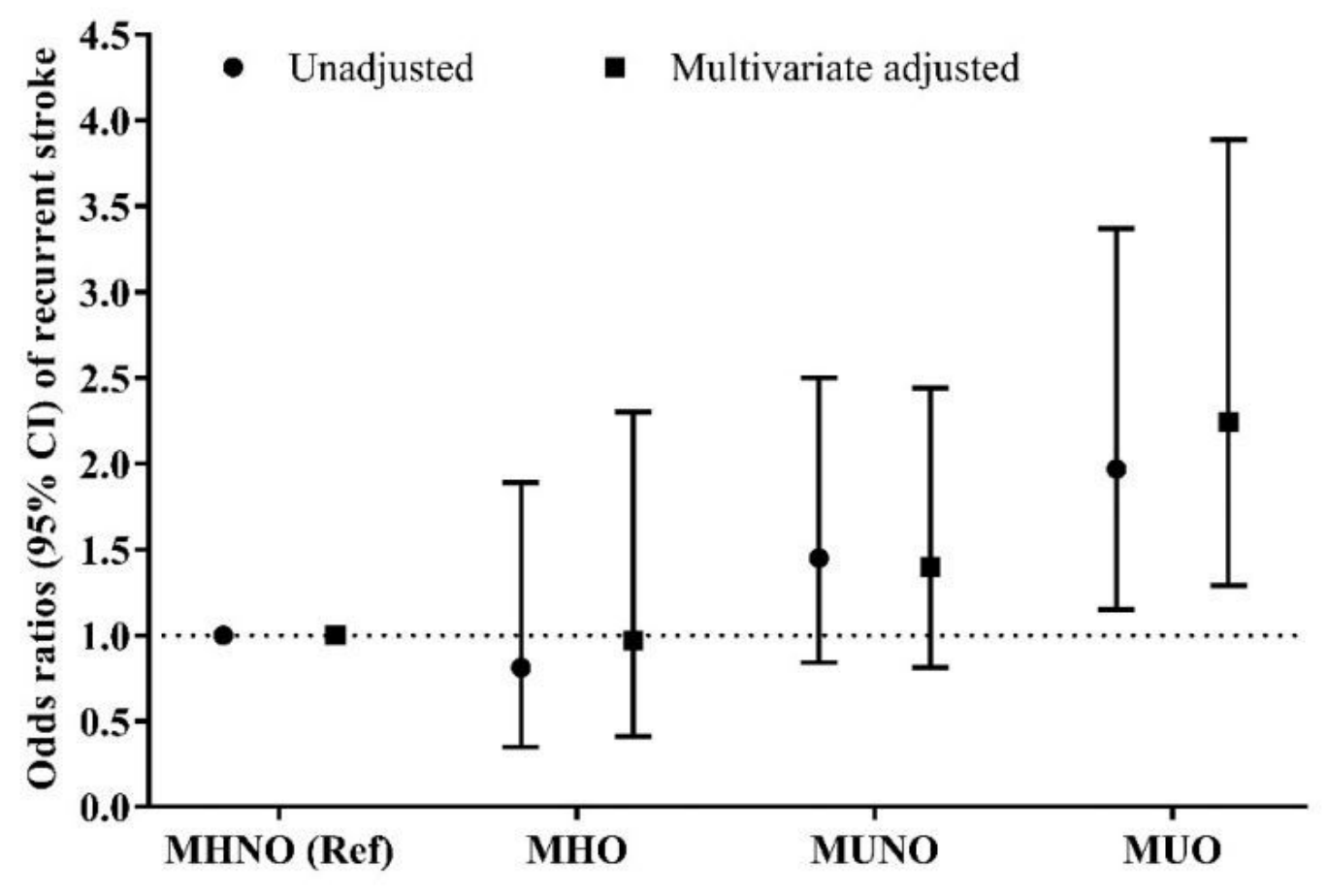

\section{Figure 1}

Risk of recurrent stroke according to metabolic and obesity phenotypes. The odds ratios of recurrent stroke were calculated using multivariate logistic regression adjusting for age, sex, current smokers (yes/no), current drinking (yes/no), physical activity, diet, education and income. Abbreviations: MHNO, metabolically healthy non-obesity; MHO, metabolically healthy obesity; MUNO, metabolically unhealthy non-obesity; MUO, metabolically unhealthy obesity. 